\title{
Finding the Number of Normal Groups in Model-Based Clustering via Constrained Likelihoods
}

\author{
Andrea Cerioli \\ Dipart. di Scienze Economiche e Aziendali, Università di Parma \\ Luis Angel García-Escudero \\ Dpto. de Estadística e I.O. and IMUVA, Universidad de Valladolid \\ Agustín Mayo-Iscar \\ Dpto. de Estadística e I.O. and IMUVA, Universidad de Valladolid \\ and \\ Marco Riani \\ Dipart. di Scienze Economiche e Aziendali, Università di Parma
}

October 5, 2018

\begin{abstract}
Deciding the number of clusters $k$ is one of the most difficult problems in cluster analysis. For this purpose, complexity-penalized likelihood approaches have been introduced in model-based clustering, such as the well known BIC and ICL criteria. However, the classification/mixture likelihoods considered in these approaches are unbounded without any constraint on the cluster scatter matrices. Constraints also prevent traditional EM and CEM algorithms from being trapped in (spurious) local maxima. Controlling the maximal ratio between the eigenvalues of the scatter matrices to be smaller than a fixed constant $c \geq 1$ is a sensible idea for setting such constraints. A new penalized likelihood criterion which takes into account the higher model complexity that a higher value of $c$ entails, is proposed. Based on this criterion, a novel and fully automated procedure, leading to a small ranked list of optimal $(k, c)$ couples is provided. A new plot called "car-bike" which provides a concise summary of the solutions is introduced. The performance of the procedure is assessed both in empirical examples and through a simulation study as a function of cluster overlap. Supplemental materials for the article are available online.
\end{abstract}

Keywords: Clustering, mixtures, EM algorithm, CEM algorithm, BIC, ICL. 


\section{Introduction}

Cluster analysis is the art of clustering a data set into $k$ groups of similar individuals. One of the main difficulties (and one of the most widely addressed problems) when using cluster analysis methods is how to decide the number of clusters $k$ to be found. Sometimes $k$ is known in advance because of the application in mind, but most of the times $k$ is completely unknown and we want the data set itself to suggest a "sensible" number of groups. Several approaches for determining the number of clusters can be found in the literature (see, e.g., Milligan and Cooper, 1985; Rousseeuw, 1987; Tibshirani et al., 2001, among many others).

In this work we tackle the problem from a model-based perspective, with a normality assumption for the cluster components. Let $x_{1}, \ldots, x_{n}$ be the observations in $\mathbb{R}^{p}$ to be clustered and let $\phi(\cdot ; \mu, \Sigma)$ denote the p.d.f. of the $p$-variate normal distribution with mean $\mu$ and covariance matrix $\Sigma$. In model based clustering, there are two distinct approaches depending on whether the mixture or the classification likelihood function is used.

The first approach is based on maximization of the mixture log-likelihood (MIX)

$$
L_{k}(\theta)=\sum_{i=1}^{n} \log \left[\sum_{j=1}^{k} p_{j} \phi\left(x_{i} ; m_{j}, S_{j}\right)\right],
$$

where $\theta=\left(p_{1}, \ldots, p_{k}, m_{1}, \ldots, m_{k}, S_{1}, \ldots, S_{k}\right)$ is the set of parameters satisfying $p_{j} \geq 0$ and $\sum_{j=1}^{k} p_{j}=1, m_{j} \in \mathbb{R}^{p}$ and $S_{j}$ a p.s.d. symmetric $p \times p$ matrix. The optimal set of parameters based on this likelihood is

$$
\widehat{\theta}_{\text {Mixt }, k}=\arg \max _{\theta} L_{k}(\theta) .
$$

Once $\widehat{\theta}_{\text {Mixt }, k}=\left(\widehat{p}_{1}, \ldots, \widehat{p}_{k}, \widehat{m}_{1}, \ldots, \widehat{m}_{k}, \widehat{S}_{1}, \ldots, \widehat{S}_{k}\right)$ is obtained, the observations in the sample are divided into $k$ clusters by using posterior probabilities. That is, observation $x_{i}$ is assigned to cluster $j$ if $j=\arg \max _{l} \widehat{p}_{l} \phi\left(x_{i} ; \widehat{m}_{l}, \widehat{S}_{l}\right)$.

The second approach is based on maximization of the classification log-likelihood (CLA)

$$
C L_{k}(\theta)=\sum_{i=1}^{n} \sum_{j=1}^{k} z_{i j}(\theta) \log \left(p_{j} \phi\left(x_{i} ; m_{j}, S_{j}\right)\right),
$$

where $\theta=\left(p_{1}, \ldots, p_{k}, m_{1}, \ldots, m_{k}, S_{1}, \ldots, S_{j}\right)$ and

$$
z_{i j}(\theta)=\left\{\begin{array}{l}
1 \text { if } j=\arg \max _{l} p_{l} \phi\left(x_{i} ; m_{l}, S_{l}\right) \\
0 \text { otherwise } .
\end{array}\right.
$$


In this case, the optimal set of parameters is

$$
\widehat{\theta}_{\text {Clas }, k}=\arg \max _{\theta} C L_{k}(\theta)
$$

and observation $x_{i}$ is now classified into cluster $j$ if $z_{i j}\left(\widehat{\theta}_{\mathrm{Clas}, k}\right)=1$.

Based on the two different likelihood approaches (1) and (2), some proposals exist that lead to sensible ways for choosing the number of clusters. The basic idea is to maximize over $k$ some complexity-penalized versions of these two likelihoods. Specifically, it is a common practice to add penalty terms depending on the number of free parameters in the model. Following this idea and taking the usual log-likelihood transformation, we envisage three possibilities:

$$
\begin{array}{ll}
\text { MIX-MIX }: & k_{\mathrm{opt}}=\arg \min _{k}\left\{-2 L_{k}\left(\widehat{\theta}_{\mathrm{Mixt}, \mathrm{k}}\right)+v_{k}\right\} \\
\text { MIX-CLA }: & k_{\mathrm{opt}}=\arg \min _{k}\left\{-2 C L_{k}\left(\widehat{\theta}_{\mathrm{Mixt}, \mathrm{k}}\right)+v_{k}\right\} \\
\text { CLA-CLA } & : \quad k_{\mathrm{opt}}=\arg \min _{k}\left\{-2 C L_{k}\left(\widehat{\theta}_{\mathrm{Clas}, \mathrm{k}}\right)+v_{k}\right\}
\end{array}
$$

where $v_{k}$ is the penalty term counting the number of free parameters. This term is typically chosen as $v_{k}=(k p+k-1+k(p+1) p / 2) \log (n)$, if no particular constraints are posed on the scatter matrices $S_{1}, \ldots, S_{k}$. In our notation, "MIX-MIX" corresponds to the use of the Bayesian Information Criterion (BIC) (see, e.g., Fraley and Raftery (2002); Fraley et al. (2017)), while "MIX-CLA" corresponds to the use of the Integrated Complete Likelihood (ICL) method proposed by Biernacki et al. (2000). The rationale behind the ICL criterion is that "mixture modeling" is a different problem from "clustering" and, thus, the number of groups obtained as a solution to these problems may not be the same. "CLA-CLA" is instead rooted in the crisp clustering framework of (2) and, to our knowledge, a novelty of this paper. The consideration of weights $p_{j}$ in classification likelihoods, as in $C L_{k}(\theta)$, goes back to Symons (1981). Bryant (1991) mentioned the possible interest in classification likelihoods with weights to choose the number of groups in clustering, but without adding an extra penalty term for model complexity. It is important to note that the maximization of the classification and mixture likelihoods are both ill-posed problems because of their unboundedness without any constraint on the scatter matrices of the fitted normal components. To overcome this problem, we impose the fulfillment of a maximal ratio constraint 
for all the eigenvalues of the scatter matrices. In addition, this eigenvalue ratio constraint is also useful to prevent traditional EM algorithms from being trapped in (non-interesting) spurious local maxima. This type of constraint depends on a fixed constant $c$ in such a way that smaller $c$ values favor cluster sphericity and homoscedasticity among groups. The use of penalized likelihood criteria under constraints, where the penalty term takes into account the higher model complexity entailed by a higher $c$ value, suggest new criteria that will be denoted as MIX $_{c}-\mathrm{MIX}, \mathrm{MIX}_{c}$-CLA and $\mathrm{CLA}_{c}$-CLA. Once the user is able to specify the type of cluster of interest through the specification of the constant $c$, these new criteria can be used to choose the number of clusters $k$. However, there are cases when that specification is not at all straightforward for the user. In those cases, as one of the main contributions of this work, we propose a fully automated procedure producing a small and ranked list of optimal $(k, c)$. All these "best ranked" solutions can be further examined, by applying validation tools in cluster analysis or taking into account the final user purposes, in order to choose the one that better fits the aims of analysis.

The outline of our work is as follows. The need for constraints in model-based clustering is reviewed in Section 2 where the maximal eigenvalue ratio constraints are also presented. Section 3 shows how well-known criteria can be adapted in this constrained setting in such a way that a "sensible" number of clusters/components can be found when the constant $c$ is fixed in advance. Section 4 addresses the important problem of choosing simultaneously both $k$ and $c$. Section 5 presents an automated procedure that returns a ranked small list of "optimal" cluster partitions and introduces a new plot which provides a concise summary of the best solutions. This procedure is illustrated in practice with both simulated and wellknown real data sets. Section 7 describes a simulation study that shows the effectiveness of the proposed methodology under general settings. Finally, Section 8 concludes and provides some open lines for future research.

\section{Constrained clustering approaches}

The need for constraints on the scatter matrices arises because both (1) and (2) are unbounded (just take $\mu_{1}=x_{1}$ and $\left|\Sigma_{1}\right| \rightarrow 0$ ). Therefore, the associated maximization becomes a mathematically ill-posed problem (see, e.g., Day, 1969). Additionally, the lack of appro- 
priate constraints often leads the algorithms proposed for numerical maximization of (1) and (2) to be trapped in local maxima of the likelihood, associated to the detection of non-interesting "spurious solutions" (see, e.g., McLachlan and Peel, 2000).

The lack of boundedness of (1) and (2) is often circumvented by resorting to "appropriate" initializations of the EM or CEM algorithms. Although this strategy is appealing, we note that, in this case, we would not be exactly trying to maximize the target functions in (1) and (2). In fact, it is known (see, e.g., Maitra (2009)) that the result of applying EM and CEM algorithms is strongly dependent on the chosen initialization, which may severely affect the value of the associated likelihoods and, consequently, the choice of $k$ provided by MIX-MIX, MIX-CLA and CLA-CLA. For instance, we may have trouble with elongated parallel clusters when using the $k$-means method, or we can be affected by undesired "chaining effects" when considering single-linkage hierarchical clustering.

Furthermore, it is important to note that cluster analysis is also not a well-defined problem from an applied viewpoint. There is nowadays a wide consensus about the fact that clustering techniques should always depend on the final data-analysis purpose, so that different goals require the use of different clustering approaches. Figure 1 in Hennig and Liao (2013) shows a toy example - to which we will return in Section 5.2.2 - with a data set obtained as a realization of a mixture of three well-separated bivariate normal components. Any clustering approach purely based on mixture modeling would determine the existence of three clusters. However, a "social stratification" framework, such as that exemplified in Hennig and Liao (2013), would clearly require the determination of more than three clusters. Similar conclusions could also hold in other important application fields, such as market research, where the construction of relevant clusters must often be coupled with subject matter aims. We thus argue that clustering should not be seen as a fully automatic task providing just one single solution and that the user always has to play an active role in it. The consideration of appropriate constraints on $\theta$, when maximizing (1) and (2), may allow the user to specify somehow the partitions of actual interest. This is another major reason that motivates our interest in introducing constraints in cluster analysis.

Some of the available solutions are based on imposing constraints on the elements of the decomposition of the scatter matrices. Different parameterizations of these scatter 
matrices allow us to obtain parsimonious variants of the unconstrained normal mixture model in such a way that (most of them) successfully serve to avoid spurious maximizers and convert the maximization of the likelihood into a well-defined problem. More details of these parsimonious parameterizations can be seen in Banfield and Raftery (1993) and Celeux and Govaert (1995). The resulting parameterizations can be easily addressed with the criteria described in Section 1 just by taking into account the number of free parameters. Another possibility, going back to Hathaway (1985), has been proposed and explored in Ingrassia and Rocci (2007) and García-Escudero et al. (2008, 2015). The approach is based on controlling the maximal ratio between the eigenvalues of the cluster scatter matrices. This implies maximizing the likelihoods (1) and (2), with $\theta \in \Theta_{c}$ where $\Theta_{c}=\left\{p_{1}, \ldots, p_{k}\right.$ with $\sum_{j=1}^{k} p_{j}=$ $1 ; m_{1}, \ldots, m_{k}$ in $\mathbb{R}^{p} ; S_{1}, \ldots, S_{k}$ p.s.d. matrices with $\lambda_{l}\left(S_{j}\right) \leq c \lambda_{q}\left(S_{h}\right)$ for every $\left.j, l, h, q\right\}$. In the above, $\left\{\lambda_{l}(S)\right\}_{l=1}^{p}$ stands for the set of eigenvalues for the scatter matrix $S$. Note that through the constant $c \geq 1$ we are simultaneously controlling discrepancies from sphericity and differences among cluster scatters. The parameter $c$ can be interpreted as the square root of the maximal ratio among the lengths of the equidensity ellipsoids defined by the $\phi\left(\cdot ; m_{j}, S_{j}\right)$ normal densities. Accordingly, we can define two constrained problems: the constrained mixture likelihood maximization $\left(\mathrm{MIX}_{c}\right)$

$$
\widehat{\theta}_{\mathrm{Mixt}, k}^{c}=\arg \max _{\theta \in \Theta_{c}} L_{k}(\theta),
$$

and the constrained classification likelihood maximization $\left(\mathrm{CLA}_{c}\right)$

$$
\widehat{\theta}_{\text {Clas }, k}^{c}=\arg \max _{\theta \in \Theta_{c}} C L_{k}(\theta) .
$$

The algorithms in Fritz et al. (2013) and in García-Escudero et al. (2014) can respectively be used to approximately solve these constrained maximizations. In these algorithms an eigenvalue truncation procedure is applied to enforce the eigenvalues ratio constraint in the EM and CEM steps. These algorithms always increase the likelihood functions throughout constrained EM and constrained CEM steps and so serve to find local maxima of the likelihood under the required constraints. Several random initializations are used when trying to find the global constrained maximum. Finally, an alternative approach can be found in Fraley and Raftery (2007) where semi-informative priors are placed on the eigenvalues of the covariance matrices. This results in a posterior mode, subsequently used in choosing the number of mixture components based on a BIC evaluated at that mode. 


\section{Penalized likelihoods in constrained clustering}

We now define the MIX $_{c}$-MIX, MIX ${ }_{c}$ CLA and CLA $_{c}$-CLA criteria for choosing the number of clusters when following the constrained maximization targets (3) and (4) for a fixed constant $c \geq 1$. This requires a modification of the "penalty term", which should take into account the higher model complexity that a higher $c$ value entails. We propose the use of a penalty term $v_{k}^{c}$ defined as

$$
v_{k}^{c}=(k p+k-1+\underbrace{k \frac{p(p-1)}{2}}_{\text {rotation par. }}+\underbrace{(k p-1)\left(1-\frac{1}{c}\right)+1}_{\text {eigenvalue par. }}) \log n .
$$

Here we have distinguished the parameters related to orthogonal rotations of the scatter matrices - which are not affected by constraints - and those related to the eigenvalues. In the most constrained case $(c=1)$, all the eigenvalues are equal, i.e. there is only one free extra parameter related to the eigenvalues. On the other hand, we recover $k p(p+1) / 2$ free parameters for the scatter matrices when we approximate the fully unconstrained case $c \rightarrow \infty$. A justification for this "soft" transition between the two extreme cases is as follows. If no constraints are posed on the whole set of eigenvalues of the scatter matrices, say $\lambda_{1}, \ldots, \lambda_{D}($ with $D=k \times p)$, then we have the reference set $A=\left\{\left(\lambda_{1}, \ldots, \lambda_{D}\right): 0 \leq \lambda_{l}\right\}$. In the

constrained case, we consider the set $B=\left\{\left(\lambda_{1}, \ldots, \lambda_{D}\right): 0 \leq \lambda_{l} \leq c \lambda_{q}\right.$ for every $\left.l \neq q\right\}$. A very simple idea is to consider the relative volume of set $B$ with respect to $A$ as a complexity measure. Of course, this ratio between volumes is not well-defined since neither $A$ nor $B$ are bounded sets. However, we can take into account that $A=\bigcup_{t \geq 0} A_{t}$ and $B=\bigcup_{t \geq 0} B_{t}$, with $A_{t}$ and $B_{t}$ being sets defined as in the statement of Theorem 3.1.

Theorem 3.1 Let $A_{t}=\left\{\left(\lambda_{1}, \ldots, \lambda_{D}\right): 0 \leq \lambda_{l} \leq t\right\}$ and $B_{t}=\left\{\left(\lambda_{1}, \ldots, \lambda_{D}\right): 0 \leq \lambda_{l} \leq\right.$ $t ; \quad \lambda_{l} \leq c \lambda_{q}$ for every $\left.l \neq q\right\}$. Then

$$
\frac{\operatorname{Vol}\left(B_{t}\right)}{\operatorname{Vol}\left(A_{t}\right)}=\left(1-\frac{1}{c}\right)^{D-1} .
$$

The proof and a graphical illustration are left to the "online supplementary material".

Theorem 3.1 is implicitly applied in our definition of the penalty term (5) by seeing that we have one "principal" eigenvalue and each of the remaining $D-1=k p-1$ eigenvalues 
are "relatively" weighted by the multiplicative factor $\left(1-\frac{1}{c}\right)$. By considering the modified penalty term $v_{k}^{c}$, we have the following three new criteria for choosing the number of clusters depending on the maximal eigenvalue ratio $c$ :

$$
\begin{aligned}
\operatorname{MIX}_{c}-\mathrm{MIX}: k_{\mathrm{opt}, \mathrm{MM}}(c) & =\arg \min _{k}\left\{-2 L_{k}\left(\widehat{\theta}_{\mathrm{Mixt}, \mathrm{k}}^{c}\right)+v_{k}^{c}\right\} \\
& :=\arg \min _{k} F_{\mathrm{MM}}(k, c) \\
\operatorname{MIX}_{c} \mathrm{CLA}: k_{\mathrm{opt}, \mathrm{MC}}(c) & =\arg \min _{k}\left\{-2 C L_{k}\left(\widehat{\theta}_{\mathrm{Mixt}, \mathrm{k}}^{c}\right)+v_{k}^{c}\right\} \\
& :=\arg \min _{k} F_{\mathrm{MC}}(k, c) \\
\mathrm{CLA}_{c}-\mathrm{CLA}: k_{\mathrm{opt}, \mathrm{CC}}(c) & =\arg \min _{k}\left\{-2 C L_{k}\left(\widehat{\theta}_{\mathrm{Clas}, \mathrm{k}}^{c}\right)+v_{k}^{c}\right\} \\
& :=\arg \min _{k} F_{\mathrm{CC}}(k, c) .
\end{aligned}
$$

Unlike the standard MIX-MIX, MIX-CLA and CLA-CLA criteria, here the use of our constrained proposals provides well-defined problems where the corresponding target functions $F_{m}(k, c), m=\mathrm{MM}, \mathrm{MC}$ or $\mathrm{CC}$, are bounded for a fixed $1 \leq c<\infty$. Moreover, spurious solutions are avoided provided that the supplied value $c$ is not very large (GarcíaEscudero et al., 2015). The specification of $c$ may be seen as a sensible way for the user to declare the maximum allowed difference on cluster scatters that he/she is willing to admit. This choice then depends on the final clustering purpose in mind. For instance, the social stratification problem in Hennig and Liao (2013) would require the user to specify a value of $c$ close to 1 . This implies the search of almost spherical clusters with similar scatters or, analogously, the use of the Euclidean distance for clustering. Other problems would require larger values of $c$, and so the detection of less restricted clusters. Once the value of $c$ has been fixed, the determination of $k_{\mathrm{opt}, m}(c)$ is done by minimizing the previously introduced criteria with respect to $k$ for a given method $m$ where $m=\mathrm{MM}, \mathrm{MC}$ or $\mathrm{CC}$. It should also be noted that this approach is not affine equivariant due to the lack of equivariance of the chosen constraints. Therefore, standardizing the variables may be needed if, for instance, very different scales are involved.

To illustrate how the methodology can be applied, let us consider a simulated data set of size $n=100$ and dimension $p=2$ from a $k=3$ component mixture obtained by applying the MixSim method of Maitra and Melnykov (2010), as extended by Riani et al. (2015) and incorporated into the FSDA toolbox of Matlab (Riani et al., 2012). The 
data set has been generated by imposing an average cluster overlap (defined as a sum of pairwise misclassification probabilities) equal to 0.04 and a maximum eigenvalue ratio for the scatters matrices equal to 5 . Given two clusters $j$ and $l(j \neq l=1, \ldots, k)$, indexed by $\phi\left(x ; m_{j}, S_{j}\right)$ and $\phi\left(x ; m_{l}, S_{l}\right)$, with probabilities of occurrence $p_{j}$ and $p_{l}$, the overlap between groups $j$ and $l$ is defined as sum of the two misclassification probabilities $w_{j l}=w_{j \mid l}+w_{l \mid j}$ where $w_{j \mid l}=\operatorname{Pr}\left[p_{l} \phi\left(x ; m_{l}, S_{l}\right)<p_{j} \phi\left(x ; m_{j}, S_{j}\right)\right]$. The average overlap is the sum of the off-diagonal elements of the matrix of the misclassification probabilities $w_{j \mid l}$ divided by $k(k-1) / 2$. Figure 1 shows two scatter plots of this simulated data set, without and with the "true" assignments labels. It is not perfectly clear by visual inspection, at least looking at the graph in the left panel, whether there are two or three clusters.
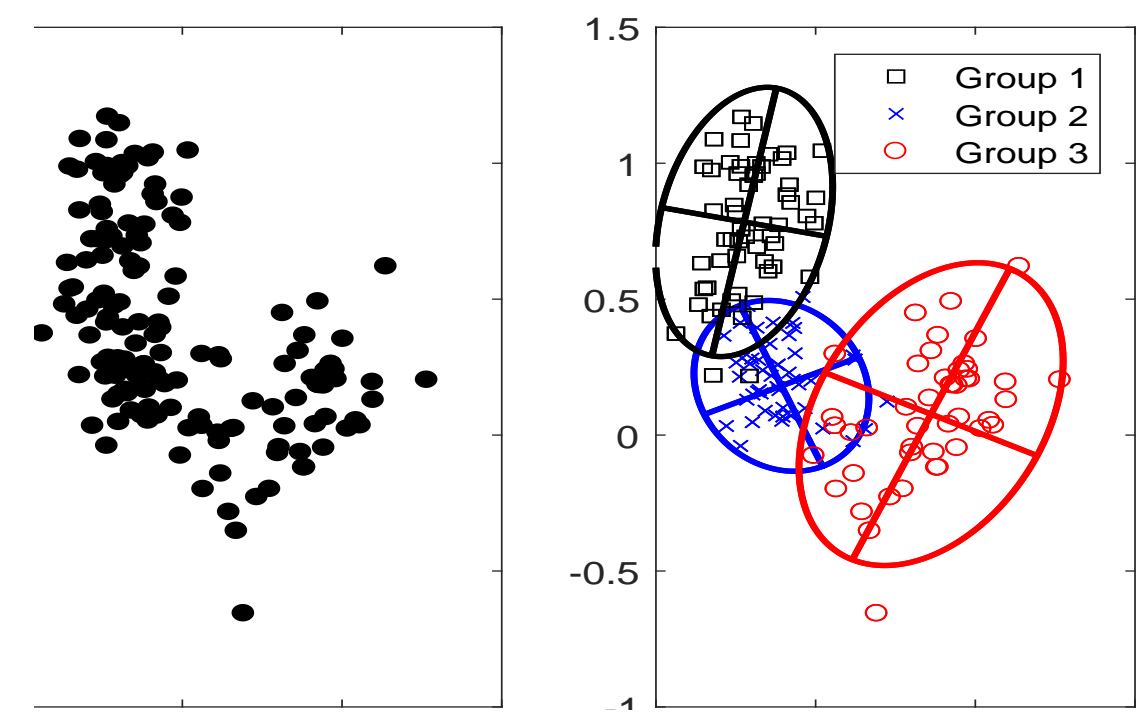

Figure 1: Simulated bivariate data set. The panel on the right shows the data set with the "true" labels and tolerance ellipsoids summarizing the three normal components.

Figure 2 shows the curves of our objective function that are obtained by monitoring $F_{\mathrm{CC}}(k, c)$ when $c$ ranges over the interval $[1,128]$ and $k$ ( $x$ axis) goes from 1 to 5 . The large left panel shows all the 8 trajectories of $F_{C C}(k, c)$ that are obtained by considering $c=\left\{2^{0}, 2^{1}, 2^{2}, \ldots, 2^{7}\right\}$. The value of $c$ for the lowest curve at each $k$ is shown in the table below the caption of the Figure. For instance, when $k=2$ the lowest value is for $c=16$; for $k=3$ the lowest value is for $c=8$; etc.. Given that the eight trajectories strongly overlap, in the first five right panels of this figure we show what happens for the five smallest values 
of $c$ we have considered $(c=1,2,4,8,16)$. The trajectories for the 3 largest values of $c$ are very similar and, thus, they are all reported in the same final right panel.
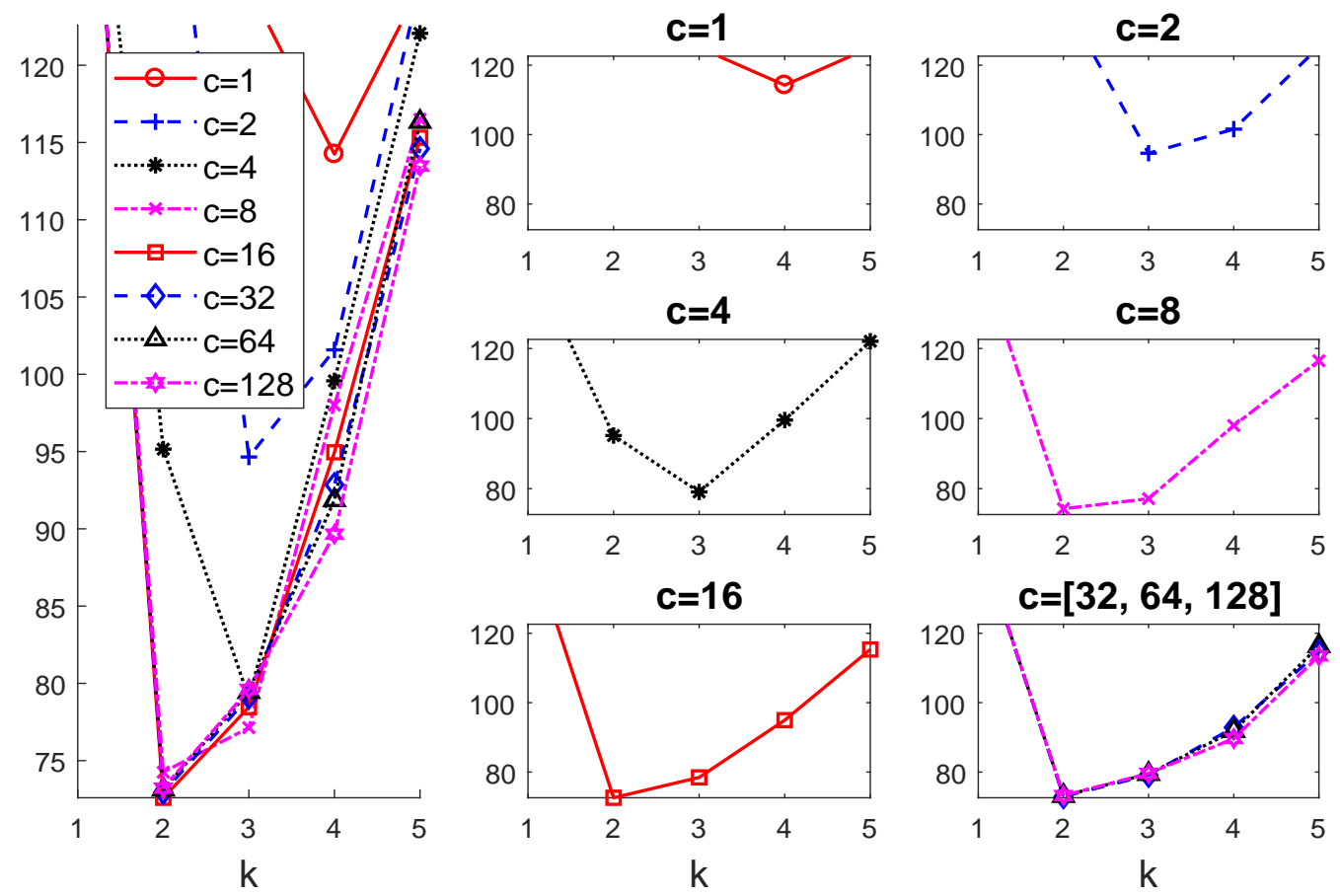

Figure 2: Analysis of the modified constained criteria when using the $\mathrm{CLA}_{c}$-CLA approach for the data set shown in Figure 1.

By using the curves plotted in Figure 2, we can see that the optimal values for the number of clusters are, for instance, $k_{\mathrm{opt}, \mathrm{CC}}(2)=3$ (i.e., when $c=2$ ) or $k_{\mathrm{opt}, \mathrm{CC}}(16)=2$ (i.e., when $c=16$ ). We thus obtain $k=3$, which corresponds to the true number of components, when we are interested in neither very spherical nor homoscedastic clusters, but we find $k=2$ clusters when we allow for more elongated group structures. The latter also provides a sensible cluster partition, from a clustering point of view, since Group 1 and Group 2 (right panel of Figure 1) may be seen as being part of the same cluster if only the left panel of Figure 1 is visualized. Similar plots are given in Figure 3 when $F_{\mathrm{MM}}(k, c)$ is monitored. We can see that the use of an objective function more focused on "mixture modeling", such as $\operatorname{MIX}_{c}$-MIX, always suggests $k_{\text {opt,MM }}(c)=3$ (i.e., the true number of mixture components) for every value of $c>1$ tried. A higher number of groups is only needed in the case $c=1$, due to the strong assumption of homoscedasticity. 

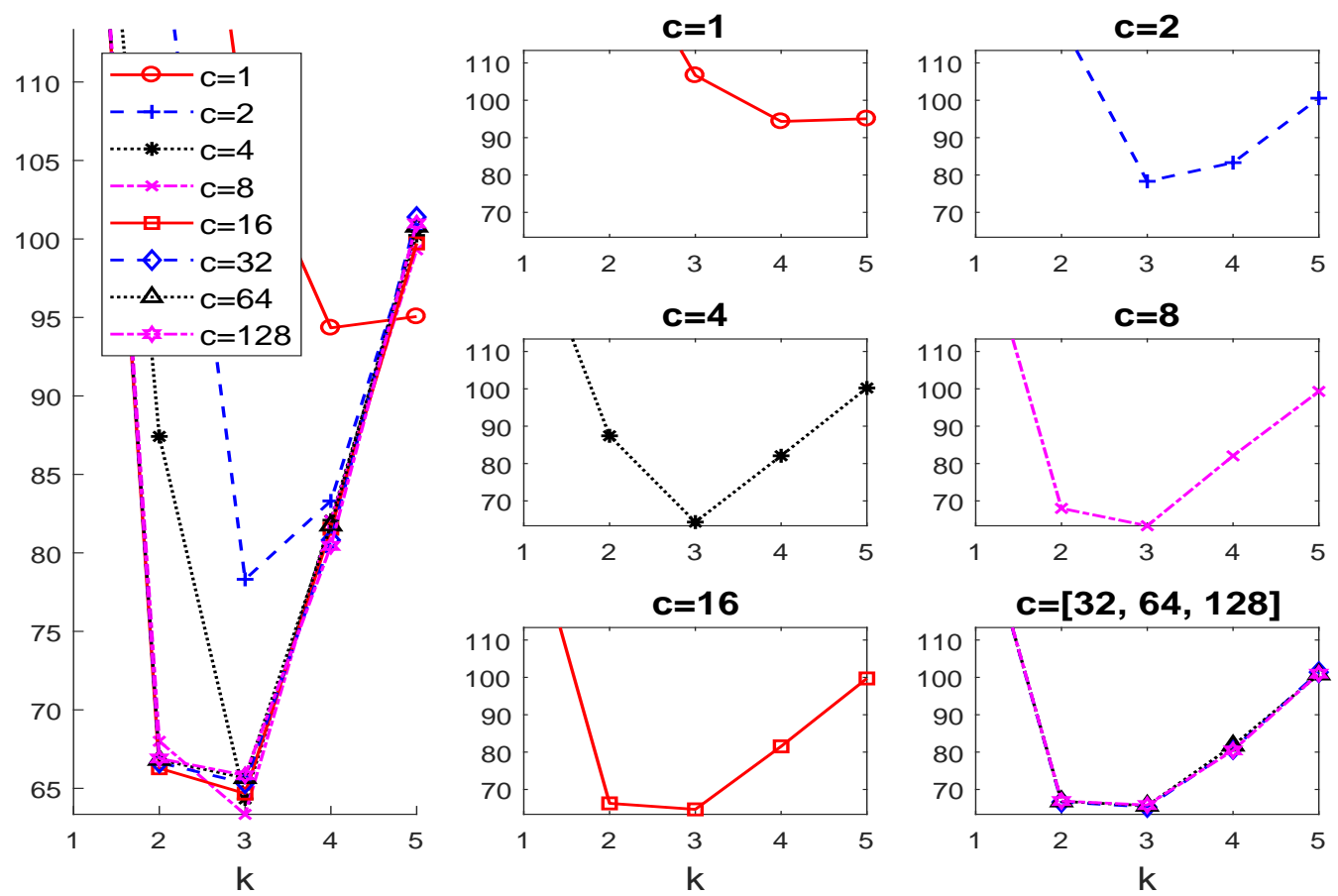

Figure 3: As Figure 2, but now for MIX $_{c}-$ MIX.

\section{Simultaneous choice of $k$ and $c$}

Alternatively, we may know the number of groups $k$ due to some economic, physical or operational reason, when our aim is that of obtaining a sensible value for $c$. Notice that in this case the user does not want to impose any particular structure on the clusters to be detected. This goal can be achieved by using the same penalized criteria as before, but now minimizing over $c$. Therefore, if $k$ is assumed to be known, we take

$$
c_{\mathrm{opt}, m}(k)=\arg \min _{c} F_{m}(k, c), \text { for } m=\mathrm{MM}, \mathrm{MC} \text { and } \mathrm{CC},
$$

as our choice for the optimal value of $c$. This information is included in the "online supplementary material" for the data set shown in Figure 1.

In practice the most interesting case is when both the proper number of clusters $k$ and the constraining factor $c$ are unknown. We have argued before that a fully unsupervised choice of both parameters, depending only on the data set at hand, is very likely to be out of reach for most applications. Nevertheless, it would be helpful if we were able to reduce the space of all possible choices of the $(k, c)$ parameter pairs to a small list of "sensible" ones, 
in order to find more easily the pair that best fits the user's main purpose in clustering.

One might think that direct study of the functionals $(k, c) \mapsto F_{m}(k, c)$, for $m=$ $\mathrm{MM}, \mathrm{MC}$ and $\mathrm{CC}$, would provide valuable information about how to choose simultaneously $k$ and $c$. Contour plots that summarize the resulting monitoring process for the data set shown in Figure 1 are in the "online supplementary material". Our experience is that these contour plots are not easily interpreted. Additionally, there are partitions obtained with different $(k, c)$ parameters that correspond to essentially the same substantial groups, or that simply differ because of the inclusion of extra (non-interesting) spurious clusters.

\section{Automated selection of a list of "sensible" solutions}

\subsection{Methodology}

In this section, we offer a fully automated procedure that leads to a small and ranked list of "optimal" choices for the pair $(k, c)$. The proposed methodology, based on our three constrained clustering criteria, relies on analysis of the stability of the cluster partitions through the Adjusted Rand Index (ARI). The ARI is a measure of the similarity between two data clusterings after "adjusting for chance". The adjusted Rand index is thus ensured to have a value close to 0 for random labeling independently of the number of clusters and samples and exactly 1 when the clusterings are identical (up to a permutation). Specifically, the procedure first detects a list with $L$ "plausible" partitions. Such "plausible" partitions may include some partitions that are essentially the same as others already detected, because spurious clusters made up with few almost collinear or very concentrated data points are found. In a second step, the partitions including spurious clusters are discarded and we end up with a (typically very) reduced and ranked list with $T$ "optimal" partitions.

Given a pair $(k, c)$, let $\mathcal{P}(k, c)$ denote the partition into $k$ subsets which is obtained by solving the problem (3) or (4), with the given $k$ and $c$ and one of the suggested methods $m=\mathrm{MM}, \mathrm{MC}$ and $\mathrm{CC}$. Let $\operatorname{ARI}(\mathcal{A}, \mathcal{B})$ denote the $\mathrm{ARI}$ between partitions $\mathcal{A}$ and $\mathcal{B}$. We consider that two partitions $\mathcal{A}$ and $\mathcal{B}$ are "essentially the same" when $\operatorname{ARI}(\mathcal{A}, \mathcal{B}) \geq \varepsilon$, for a fixed threshold $\varepsilon$. Clearly, the higher the value of the threshold the greater is the number of tentative different solutions which are considered. 
Let us consider the sequence $k=1, \ldots, K$, where $K$ is the maximal number of clusters, and a sequence $c=c_{1}, \ldots, c_{C}$ of $C$ possible constraint values. For instance, the sequence of powers of $2, c_{1}=2^{0}, c_{2}=2^{1}, \ldots, c_{C}=2^{C-1}$ is recommended because it enables us to consider a sharp grid of values close to 1 . By using this notation, the proposed automated procedure may be described as follows:

1. Obtain the list of "plausible" solutions:

1.1 Initialize: Start with $K \times C$ possible $(k, c)$ pairs to be explored. Let $\mathcal{E}_{0}=\{(k, c)$ : $k=1, \ldots, K$ and $\left.c=c_{1}, \ldots, c_{C}\right\}$.

1.2 Iterate: If $\mathcal{E}_{l-1}$ is the set of pairs $(k, c)$ not already explored at stage $l-1$, then:

1.2.1 Obtain $\left(k_{*}^{l}, c_{*}^{l}\right)=\arg \min _{(k, c) \in \mathcal{E}_{l-1}} F_{m}(k, c)$.

1.2.2 Remove all of the cluster partitions $(k, c) \in \mathcal{E}_{l-1}$ with $k=k_{*}^{l}$ and values of $c$ which are adjacent to $c_{*}^{l}$, and such that they are very "similar" to partition $\mathcal{P}\left(k_{*}^{l}, c_{*}^{l}\right)$ for the given threshold value $\varepsilon$, in the sense that

$$
\operatorname{ARI}\left(\mathcal{P}(k, c), \mathcal{P}\left(k_{*}^{l}, c_{*}^{l}\right)\right) \geq \varepsilon
$$

Take $\mathcal{E}_{l}$ as the set $\mathcal{E}_{l-1}$ after removing the pairs yielding "similar" partitions.

1.3 Finalize: The iterative procedure ends when $\mathcal{E}_{L}=\emptyset$ (or when $L$ is a positive prefixed integer number) and it returns $\left\{\left(k_{*}^{1}, c_{*}^{1}\right),\left(k_{*}^{2}, c_{*}^{2}\right), \ldots,\left(k_{*}^{L}, c_{*}^{L}\right)\right\}$ as a list with $L$ "feasible" parameters combinations.

2. Obtain the list of "optimal" solutions:

2.1 Initialize: Start from $\mathcal{I}_{0}=\{1, \ldots, L\}$ and the $L \times L$ matrix $\left(d_{r, s}\right)_{r, s=1, \ldots, L}$, where

$$
d_{r, s}=\operatorname{ARI}\left(\mathcal{P}\left(k_{*}^{r}, c_{*}^{r}\right), \mathcal{P}\left(k_{*}^{s}, c_{*}^{s}\right) .\right),
$$

2.2 Iterate: Given $\mathcal{I}_{t-1}$ the non discarded "plausible" solutions at stage $t-1$ :

2.2.1 Take $\left(k_{\mathrm{opt}}^{t}, c_{\mathrm{opt}}^{t}\right)=\left(k_{*}^{l_{t}}, c_{*}^{l_{t}}\right)$ where $l_{t}$ is the $t$-th element of $\mathcal{I}_{t-1}$ (where the indexes in $\mathcal{I}_{t-1}$ are sorted from lowest to highest).

2.2.2 Discard "spurious" solutions (i.e., those that are similar to the already detected "optimal" ones): $\mathcal{I}_{t}=\mathcal{I}_{t-1} \backslash\left\{r: r \in \mathcal{I}_{t-1}, r>l_{t}\right.$ and $\left.d_{r, l_{t}} \geq \varepsilon\right\}$. 
2.3 Finalize: The iterative procedure ends when $\mathcal{I}_{T}=\emptyset$. It returns

$$
\left\{\left(k_{\mathrm{opt}}^{1}, c_{\mathrm{opt}}^{1}\right),\left(k_{\mathrm{opt}}^{2}, c_{\mathrm{opt}}^{2}\right), \ldots,\left(k_{\mathrm{opt}}^{T}, c_{\mathrm{opt}}^{T}\right)\right\}
$$

as the "optimal" pairs.

To simplify notation, we have deleted the subscript $m$ for the criterion used (i.e., $\left(k_{\mathrm{opt}}^{t}, c_{\mathrm{opt}}^{t}\right)$ should be $\left(k_{\mathrm{opt}, m}^{t}, c_{\mathrm{opt}, m}^{t}\right)$ for $m=\mathrm{MM}, \mathrm{MC}$ and $\left.\mathrm{CC}\right)$. Additionally, the complete automated procedure is hereinafter referred to autMIXMIX, autMIXCLA and autCLACLA.

For each "optimal" pair $\left(k_{\mathrm{opt}}^{t}, c_{\mathrm{opt}}^{t}\right)$, it is also informative to take into account the socalled "best interval" $\mathcal{B}_{t}$ defined as

$$
\mathcal{B}_{t}=\left\{c: F_{m}\left(k_{\mathrm{opt}}^{t}, c_{\mathrm{opt}}^{t}\right) \leq F_{m}\left(k_{\mathrm{opt}}^{t}, c\right)\right\}
$$

and the so-called "stable interval" defined as

$$
\mathcal{S}_{t}=\left\{c: \operatorname{ARI}\left(\mathcal{P}\left(k_{\mathrm{opt}}^{t}, c\right), \mathcal{P}\left(k_{\mathrm{opt}}^{t}, c_{\mathrm{opt}}^{t}\right)\right) \geq \varepsilon\right\}
$$

A large interval $\mathcal{B}_{t}$ means that the number of clusters $k_{\text {opt }}^{t}$ is "optimal", in the sense of (7), for a wide range of $c$ values. A large interval $\mathcal{S}_{t}$ means that the solution is "stable", in the sense of (8), because it does not essentially change when moving $c$ in that interval.

\subsection{Examples}

Three examples have been included in the text to exemplify the performance of the proposed automated procedure. The first has to do with its application to the previously simulated data set and the second one considers a "toy example" which serves to illustrate how different cluster partitions may be needed depending on the final clustering purposes. The third example is based on a more complex "oil data set" with $p=8$ variables and a possible larger number of clusters $k$. The "online supplementary material" includes an additional example when the methodology is applied for the classical and well-known "Iris data set".

\subsubsection{Application to simulated data}

We have applied the proposed automated procedure with an ARI to the simulated data set displayed in Figure 1. We start with the classification approach and the needed tables to 
reproduce the analysis displayed in this section can be seen in the "online supplementary

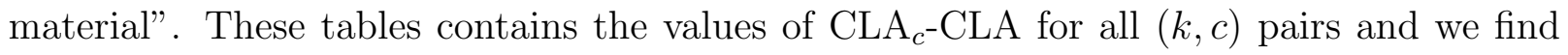
the threshold given in equation (6) considering the matrix which contains the ARI indexes for two consecutive values of $c$ given $k$. The first table there shows that (as anticipated in the previous section) the best value of $k$ is 2 and the second best value of $k$ is 3 . The second table there, on the other hand, shows that for $k=2$ (overall best solution) there is essentially just one solution in the range $c[2,128]$ (the values of ARI in this interval are all greater than 0.8 ). For $k=3$ (second overall best solution) the solution is stable in the interval $c[1,128]$ with many solutions giving exactly the same partition. The situation seems to be more complex for $k=4$ where in the interval $c[16,128]$ we virtually obtain the same solution. The ARI index between $c=8$ and $c=16$ when $k=4$ is 0.71 and suggests a moderate change in the classification, while the one between $c=4$ and $c=8$ goes down to 0.57 . Finally, the solutions in the interval $c[1,4]$ seem homogeneous. Our procedure combines in a fully automatic way the information in the two previous tables. The analysis of the the values of $\mathrm{CLA}_{c^{-}}$CLA for all $(k, c)$ pairs shows that the first best solution is for $k=2$ and $c=16$ and that this solution remains the best in the interval $c[8,128]$ and (if we consider a threshold of ARI equal to 0.8) we find that this solution is stable in the interval $c[2,128]$. In order to find the second solution, we remove from the table with all the values of $\mathrm{CLA}_{c}$-CLA those $(k, c)$ pairs in which the first solution was stable and check for the new minimum. The joint examination of the two tables, shows that the second solution takes place when $k=3$, and $c=8$, and that this solution is best and stable in the interval $c$ $[4,128]$ and that is stable in the interval $c[1,128]$. Given that the ARI index between the second solution $(k=3$ and $c=8)$ and the first one $(k=2$ and $c=16)$ is 0.38 (below the prefixed threshold of 0.8 we consider the second solution as non spurious). The procedure goes on until we find the the first (say) three or four non spurious solutions.

The corresponding four best-ranked solutions are shown in Figure 4. We see that we recover the true number of clusters $k_{\mathrm{opt}, \mathrm{CC}}^{2}=3$ in the second solution. The solution with $k_{\mathrm{opt}, \mathrm{CC}}^{1}=2$ makes perfect sense from the "pure" clustering point of view adopted by the CLA $_{c}$-CLA criterion and, thus, it is the first offered partition. The homoscedastic $c=1$ solution is shown as the ninth one and it proposes $k_{\mathrm{opt}, \mathrm{CC}}^{9}=5$ clusters. 

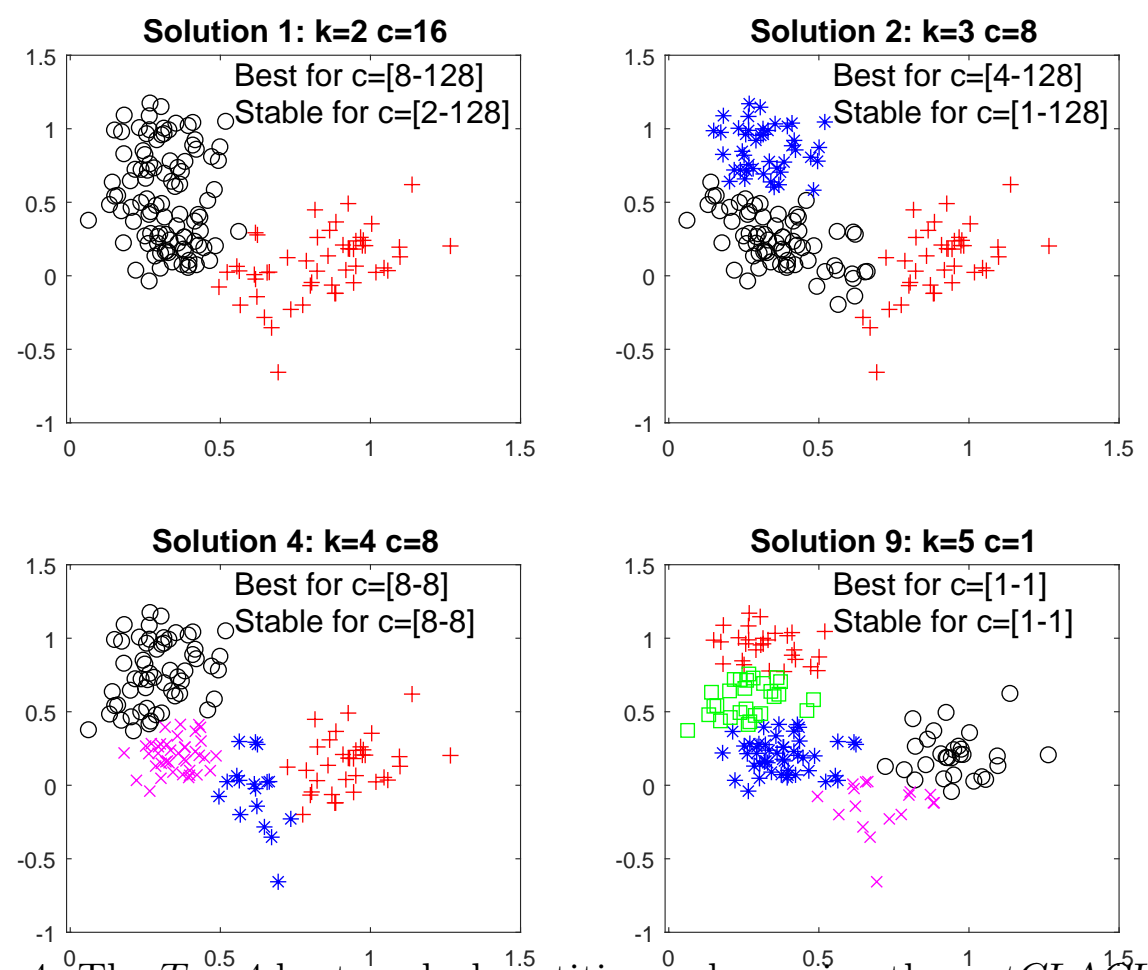

Figure $4:^{0}$ The $T \stackrel{0.5}{=} 4$ best-ranked partitions when ${ }^{1.5}{ }^{-1}{ }^{0}$ ing the ${ }^{1}$ autCLA ${ }^{15} L A$ procedure for the simulated data set displayed in Figure 1.

In order to provide a concise summary of the non spurious solutions found and in order to better distinguish the most relevant solutions from those which are local, we propose a new graphical display which we call "car-bike". This plot shows on the horizontal axis the value of $c$ and on the vertical axis the value of $k$. For each solution we draw a rectangle for the interval of values for which the solution is best and stable and a horizontal line which departs from the rectangle for the values of $c$ in which the solution is only stable. Finally, for the best value of $c$ associated to the solution, we show a circle with two numbers, the first number indicates the ranked solution among those which are not spurious and the second one the ranked number including the spurious solutions. This plot has been baptized "car-bike", because the first best ranked solutions (in general 2 or 3) are generally best and stable for a large number of values of $c$ and therefore will have large rectangles. In addition, these solutions are likely to be stable for additional values of $c$ and therefore are likely to have horizontal lines departing from the rectangles (from here the name "cars"). 
Finally, local minor solutions (which are associated with particular values of $c$ and $k$ ) do not generally present rectangles or lines and are shown with circles (from here the name "bikes"). Figure 5 shows the car-bike plot coming from the autCLACLA procedure. This plot shows that there are two main solutions ("cars") one with 2 groups and the second with 3 groups. In this case, the two cars are station wagons because the horizontal line just departs from the left side of the rectangles. The other two non-spurious solutions ("bikes") are for $k=4$ and $c=8$, and $k=5$ and $c=1$. An additional bonus of this new graphical representation is that it allows us to immediately spot the area where the best values of $c$ lie. If we examine the car-bike plot from a vertical perspective we can see that the optimal values of $c$ are concentrated in the zone between $c=8$ and $c=16$. In the car-bike plot, the height of the rectangles can be made proportional to the order of the ranked solution. Using this criterion the height of the rectangle of say solution 3 out of 8 is $(8-3) / 8$-th that of the first. Careful examination of Figure 5 reveals that the height of the rectangle for the second best ranked solution is slightly smaller than that of the first one.

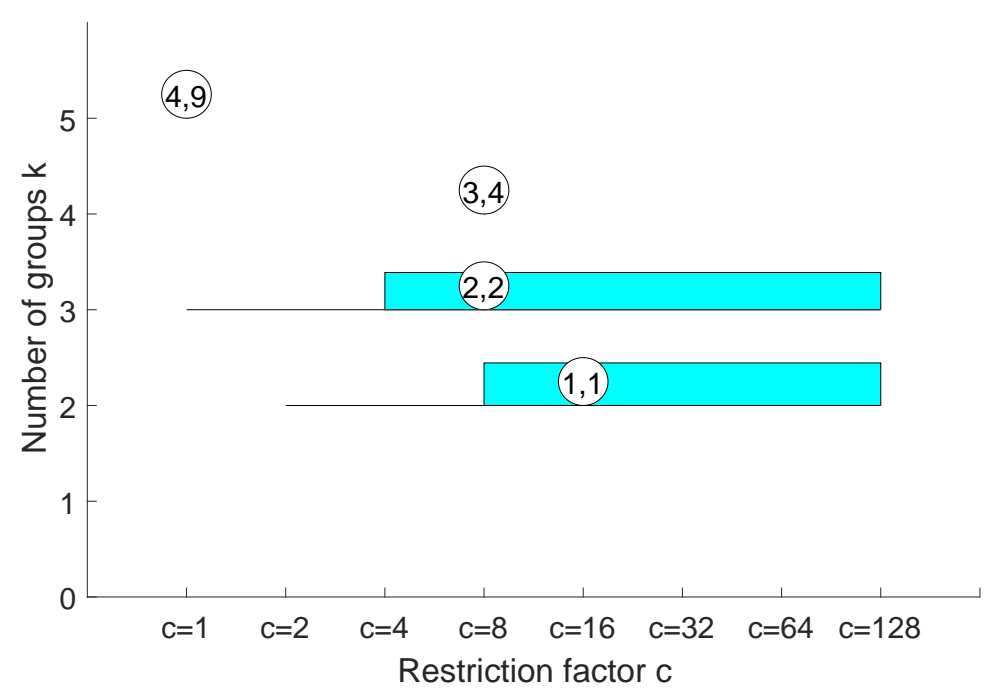

Figure 5: The "car-bike" plot when using using the autCLACLA procedure for the simulated data set displayed in Figure 1.

A figure showing the first 4 discarded "spurious" solutions for the simulated data set can be found in the "online supplementary material". We can see there that these discarded solutions either include clusters made up with a few almost collinear or concentrated ob- 
servations or correspond to solutions close to one already detected "optimal" partition.

A referee suggested us to comment on the choice of the threshold of the ARI index. Our experience is that the value of the threshold is important just to highlight/hide the local solutions "bikes", but plays no rule in the detection of the main solutions ("cars"). In the example above, we have used a threshold of the Rand index equal to 0.8. If we consider a threshold of 0.7 the only modification concerns the third non spurious solution (bike associated with $k=4$ and $c=8)$ which disappears because the third solution $(c=128$ and $k=4$ which is spurious and therefore is not pictured in the plot) with the new threshold is considered stable in the interval $c[8,128]$ (as seen in the "online supplementary material").

Figure 6 shows the ranked set of "optimal" solutions when using the autMIXMIX procedure. Notice that, from a mixture modeling point of view, we obtain the correct

number of components $\left(k_{\mathrm{opt}, \mathrm{MM}}^{1}=3\right)$ in the first position. This result agrees with the well known fact that mixture modeling is better suited to address cluster overlap than "pure" clustering, which instead ideally assumes well-separated clusters. The car-bike plot (not given here for lack of space) for autMIXMIX shows two big cars and and some bikes scattered around showing once again the presence of just two relevant solutions.

\subsubsection{Application to Hennig and Liao's type of data}

Section 5 in Hennig and Liao (2013) includes a toy example to illustrate that there are cases "where a mixture model is true and most people may have a natural intuition about the true clusters" but these clusters "are not necessarily the clusters that a researcher is interested in". In the spirit of that toy example, we consider the simulated data set shown in Figure 7. This data set corresponds to a realization of a mixture of three well-separated bivariate normal components. Without knowledge of the underlying substantive problem, one would then agree that $k=3$ is a sensible choice for $k$. However, let us assume (as Hennig and Liao did) that we are facing a social stratification clustering problem and that the two variables are, for instance, an income and a status indicator. By choosing $k=3$ and very unrestricted scatter matrices, one cluster would contain both the poorest people with lowest status and the richest people with the highest status. Therefore, in this particular application, a higher number of (more homoscedastic) clusters is surely needed. 

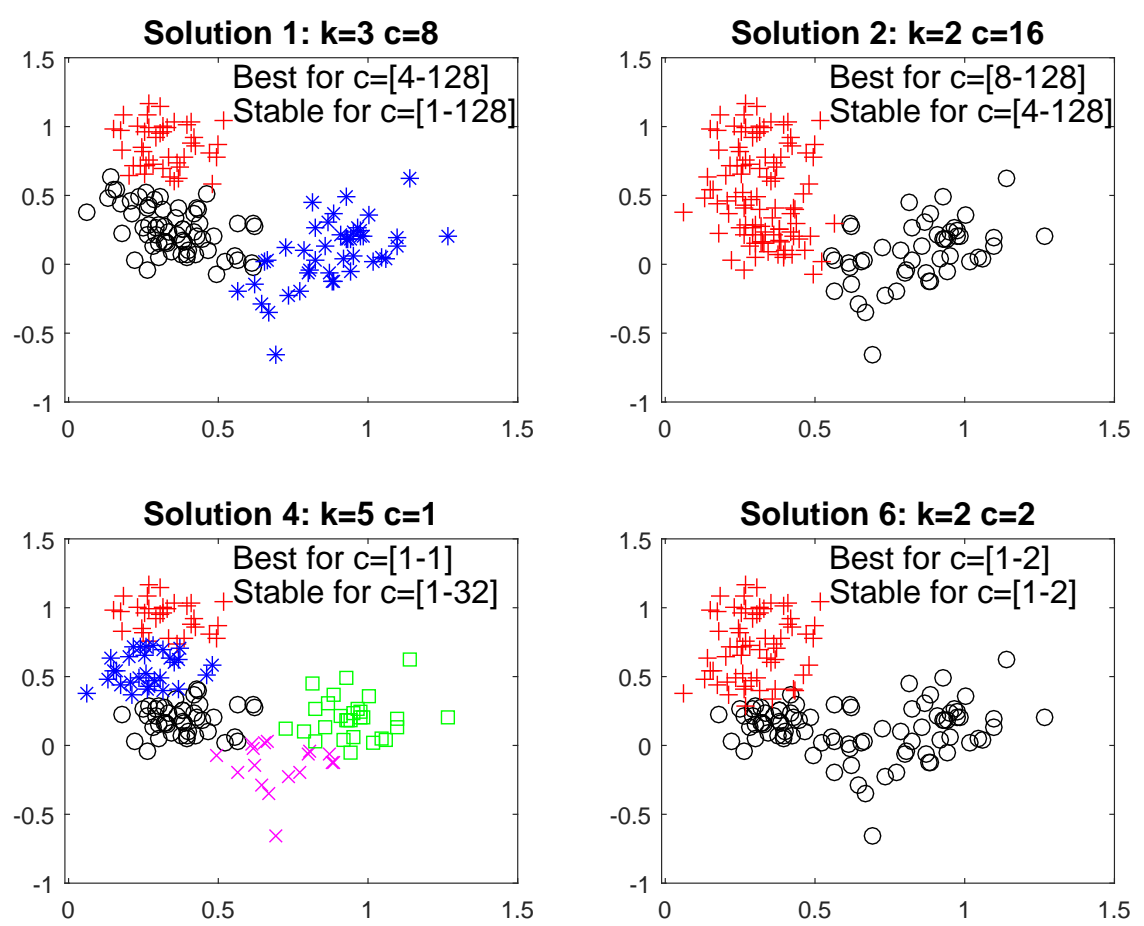

Figure 6: The $T=4$ "optimal" partitions when using the autMIXMIX procedure for the data set displayed in Figure 1.

Figure 7 shows $T=4$ "optimal" solutions when using the autMIXMIX procedure. We can see that the best-ranked partition is exactly the one which discovers the 3 bivariate normal components. The second and third best ranked partitions offer the user a more sensible clustering partition for that particular "social stratification" problem. The fourth best ranked solution offers a very peculiar partition where the two more concentrated normal components are surprisingly joined together. However, this more "exotic" solution just appears after the more "sensible" ones. In any case, we think that it is useful to reduce all the possible pairs $(k, c)$ to such a type of small lists of best-ranked partitions, where the user can hopefully choose the one that better fits his/her clustering purposes. The car-bike plot for this data set is presented in the "online supplementary material".

\subsubsection{Olive oil data set}

In the previous examples we have seen how our methodology works in the presence of a small number of clusters. The purpose of this section is to test our proposal on a more real 

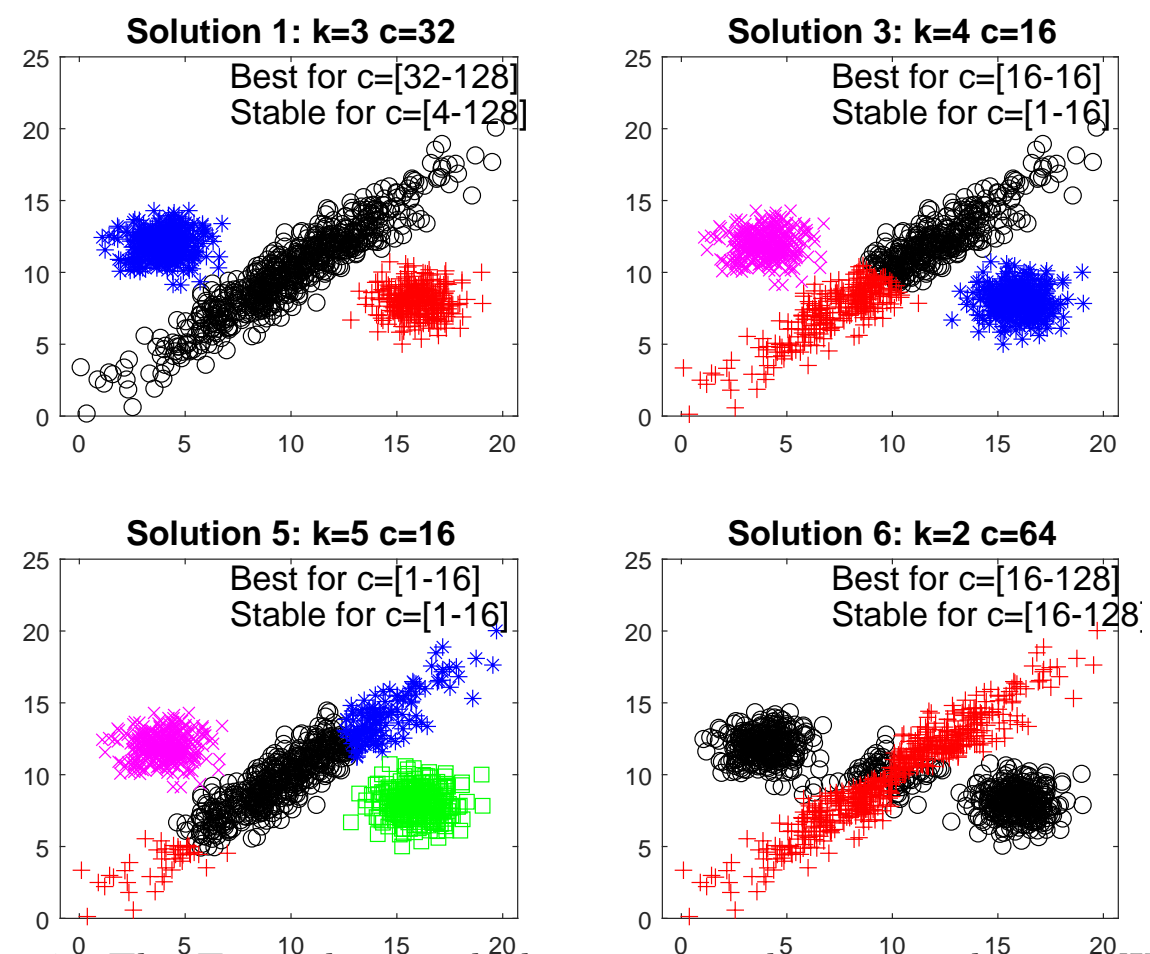

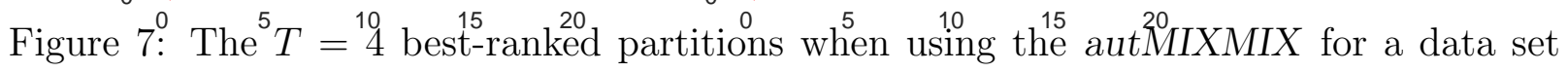
similar to that in Hennig and Liao (2013).

and complex dataset which contains several 'possibly' overlapping groups.

The "olive oil" data set (Forina et al., 1983) contains $p=8$ chemical measurements on the acid components of $n=572$ olive oil specimen produced in various regions in Italy: (1) North Apulia, (2) Calabria, (3) South Apulia, (4) Sicily, (5) Inland Sardinia, (6) Costal Sardinia, (7) East Liguria, (8) West Liguria, and (9) Umbria. This data set is available, for instance, from the pgmm package at CRAN (McNicholas et al., 2015). We standardize these variables and apply the autMIXMIX criterion with a maximal number of clusters $K=12$ and tentative constraining factors $c=1,2,4, \ldots, 128$. The three best ranked solutions obtained are $(c=128, k=6),(c=128, k=7)$ and $(c=128, k=5)$. The use of the mclust package (Fraley and Raftery, 2002; Fraley et al., 2017) returns the following three best ranked models: (VVV, $k=5$ ), (VVV, $k=9$ ) and (VVV, $k=8$ ) which are obtained by using the BIC criterion. "VVV" stands for ellipsoidal and varying volume, shape, and orientations. This is in concordance with the choice $c=128$, i.e. scatter matrices as unconstrained as possible. Table 1 shows the ARI values obtained for the three 
Table 1: ARI values of the 3 best ranked solutions with respect to the true regions in the "Olive oil" dataset. The proposed models appear within parenthesis.

\begin{tabular}{|llll|}
\hline & \multicolumn{3}{c|}{ ARI values } \\
\cline { 2 - 4 } & First & Second & Third \\
\hline autMIXMIX & $0.8060(c=128, k=6)$ & $0.8468(c=128, k=7)$ & $0.7441(c=128, k=5)$ \\
mclust & $0.7763(\mathrm{VVV}, k=5)$ & $0.6258(\mathrm{VVV}, k=9)$ & $0.6601(\mathrm{VVV}, k=8)$ \\
\hline
\end{tabular}

best ranked solutions with respect to the true classification in the 9 regions.

In this case the three best partitions returned by autMIXCLA and autCLACLA are essentially the same (and in the same order) in this case.

Table 2 shows the confusion matrix for the second best solution (that with ARI value 0.8468). We can see the original oil production regions are quite well recovered in this suggested cluster partition. The only regions that we are not able to disentangle are regions 2 and 4 (Calabria and Sicily) and regions 5 and 6 (inland and costal Sardinia). A thorough examination of the scatter plot matrix reveals that oils from regions 2 and 4 take values on these fatty acid contents that are almost virtually impossible to distinguish. Regions 5 and 6, apart from belonging to same island, can only be slightly discriminated in just two out of the eight variables. Additionally, regions 5 and 6 constitute a rather compact and well-differentiated cluster with respect the other regions when joined together. Although the use of the traditional BIC criterion within mclust sometimes suggests $k=9$ clusters, the partition proposed with this number of clusters is far from the true one, mainly because the largest "South Apulia" cluster is split into two clusters.

\section{Example: Road traffic data}

In this example, we illustrate the proposed methodology in a road traffic problem. The data set comes from speed measurements collected in an expressway in Paris. To be more precise, data are from a fixed station that catches the average speed of cars passing through it resulting in 180 daily measurements from 5:00 a.m. to 23:00 p.m. This data set was used in García-Escudero and Gordaliza (2005) to illustrate the performance of a robust functional 
Table 2: Confusion matrix for the second best partition obtained when using the autMIXMIX method.

\begin{tabular}{|lccccccc|}
\hline Regions \Clusters & 1 & 2 & 3 & 4 & 5 & 6 & 7 \\
\hline 1. North Apulia & 0 & 24 & 0 & 0 & 1 & 0 & 0 \\
2. Calabria & 0 & 0 & 0 & 0 & 55 & 0 & 1 \\
3. South Apulia & 0 & 0 & 0 & 0 & 7 & 0 & 199 \\
4. Sicily & 0 & 18 & 0 & 0 & 16 & 0 & 2 \\
5. Inland Sardinia & 0 & 0 & 0 & 65 & 0 & 0 & 0 \\
6. Costal Sardinia & 0 & 0 & 0 & 33 & 0 & 0 & 0 \\
7. East Liguria & 12 & 0 & 38 & 0 & 0 & 0 & 0 \\
8. West Liguria & 50 & 0 & 0 & 0 & 0 & 0 & 0 \\
9. Umbria & 0 & 0 & 3 & 0 & 0 & 48 & 0 \\
\hline
\end{tabular}

clustering methodology. The highly rough speed curves were smoothed by projection onto a basis of cubic B-splines resulting from using 6 equispaced knots. Thus, each of these curves is converted into a $p=10$ dimensional vector. Although we start with data corresponding to 617 consecutive days, we only consider 493 curves surviving the trimming approach described in García-Escudero and Gordaliza (2005). Anomalous traffic days with slow traffic during very discontinuous and large time-periods, or days when the speed detector seems to provide wrong measurements, are discarded by using this trimming approach.

When applying the proposed methodology to this $n \times p=493 \times 10$ data set with a maximal number of clusters $K=10$ and constraining factors $c=1,2,4, \ldots, 128$, the three best ranked partitions are obtained for $k=3, k=4$ and $k=2$ (in this order) and $c=128$. The proposed clustering partition is exactly the same regardless of whether the autMIXMIX or autCLACLA approach are used. The three best ranked solutions are presented in the "online supplementary material" by using the functional boxplots of Sun and Genton (2011). The first proposed solution detects 3 clusters: the first cluster is made up of days where speed remains almost constant and high throughout the whole day, the second day includes days where the average speed notably decreases in the evening and, finally, the third cluster contains those days where speed does not decrease in the evening, 
but this decrease seems to happen in the morning. The second solution offered is similar to the first one but a fourth cluster is additionally detected. This fourth cluster includes days where the speed is not very high at any moment of the day and traffic jams arise both in the morning and in the evening. Finally, the third best ranked solution detects two clusters where the second one seems to include the days where the speed decreases in the evening.

\section{$7 \quad$ Simulation study}

The purpose of this section is to analyze the performance of the autMIXMIX, autMIXCLA and autCLACLA procedures as a function of the overlap between the groups. We have considered an example with clusters with true number of groups equal to 3 , true eigenvalue ratio equal to $6, n=150$, and an average overlap which goes from 0.01 to 0.1 , with step 0.01. We have performed 100 simulations for each setting in dimensions $p=2$ and 6 . In each simulation, with the aim of "visiting" as many as possible different $\theta$ vectors, we have considered several random initializations (nstarts=1000) obtained from drawing $k \times(p+1)$ observations that are arranged into $k$ groups with $p+1$ observations. By using these $k$ groups, we obtain $k$ initial $m_{j}$ centers through their sample means and $k$ initial scatter parameters $S_{j}$ through their sample covariance matrices. In order to start with an initial admissible solution we have immediately applied the eigenvalue constraint. The values of $c$ which are considered go from 1 to $128\left(c=\left\{2^{0}, 2^{1}, 2^{2}, \ldots, 2^{7}\right\}\right)$ and the values of $k$ go from 1 to 5 . In order to avoid the randomness due to different starting points, both for mixture and classification likelihoods, for each simulation we have considered the same 1000 initial subsets for each value of $c$. For each simulation and each procedure, we have stored:

1. The ARI between the true solution and the best-ranked solution found automatically;

2. The maximum ARI value between the true solution and the first two best-ranked solutions found automatically;

3. The maximum ARI value between the true solution and the first three best-ranked solutions found automatically. 

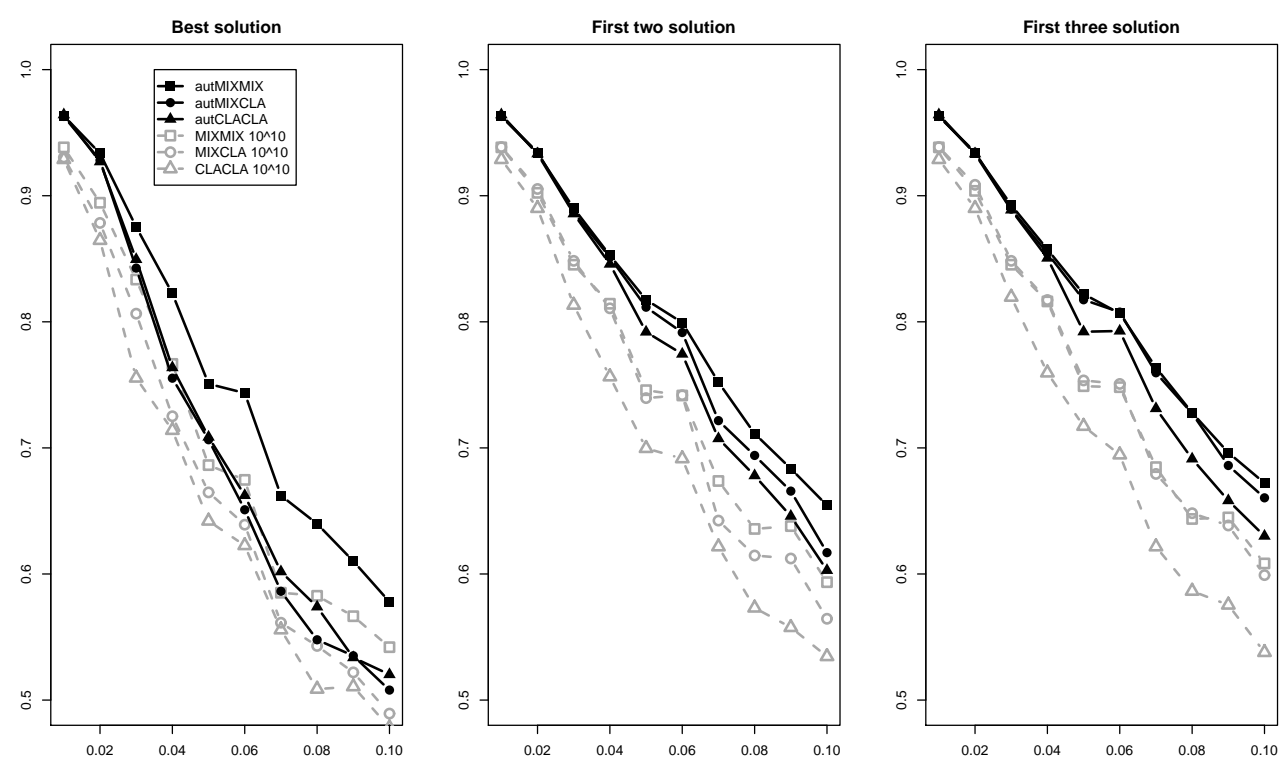

Figure 8: Average ARI index across 100 simulations as a function of cluster overlap when $p=2$. The ARI indexes between the true solution and the best solution are shown in the left panel; with respect to the first two best-ranked solutions in the central panel and with respect to first three best-ranked ones in the right panel. The results of applying "traditional" ICL and BIC criteria (i.e., the use of MIX-MIX and MIX-CLA almost unconstrained with $c=10^{10}$ ) are shown in grey.
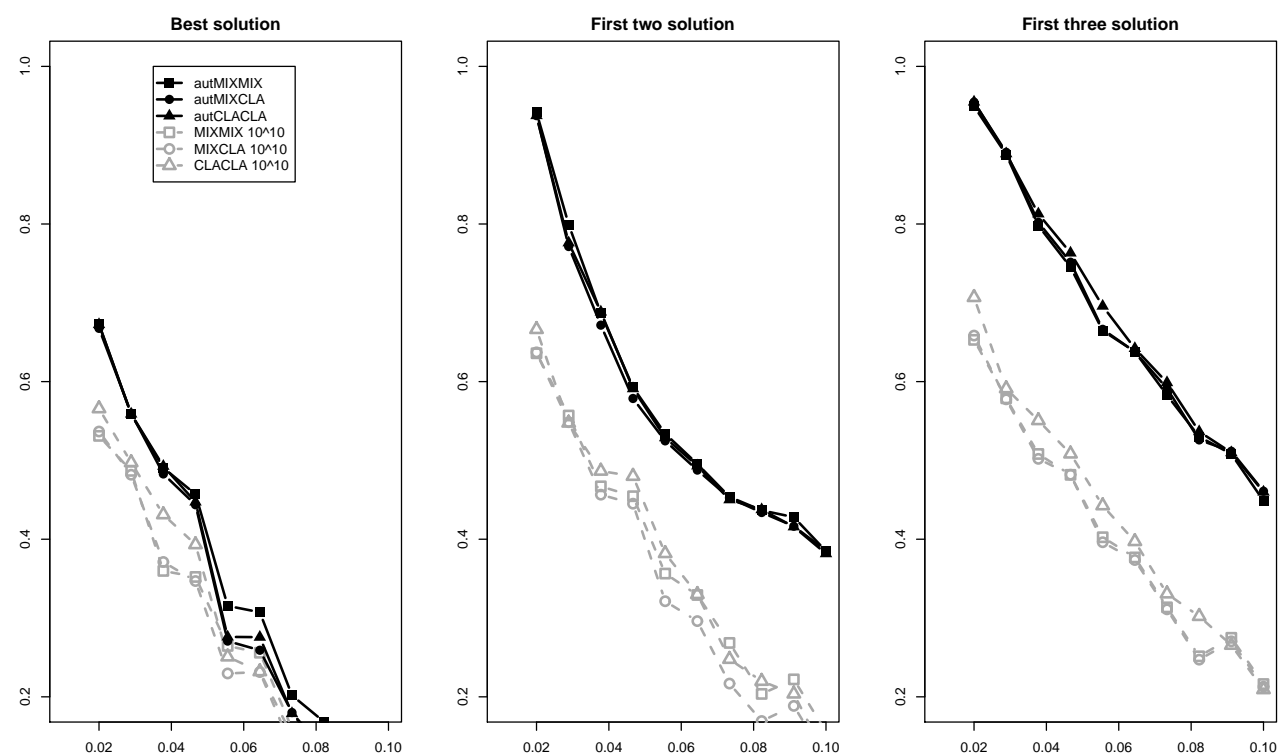

Figure 9: As Figure 8, but now with $p=6$. 
Figure 8 shows the average values of the above ARI over 100 simulations when the dimension of the simulated data set is $p=2$. The left panel of the figure shows that as the average overlap increases the best performance is for the autMIXMIX procedure. More precisely, if the overlap is small the 3 information criteria give equivalent results. On the other hand, as the overlap increases, the gap between autMIXMIX and the other two information criteria increases. When we consider just the first solution, the curves for autMIXCLA and autCLACLA are virtually the same when the average overlap is smaller than 0.04 but the curve associated with autMIXCLA seems to be slightly higher than that of autCLACLA for high values of overlap. When we consider the first two solutions, the curve of autMIXCLA is always in between autMIXMIX and autCLACLA. Finally, when we consider the first three best solutions the curve of autMIXCLA is virtually equal to that of autMIXMIX, even if autMIXMIX still prevails for large overlap.

In order to show the effect of constraints, in Figure 8, we have also added the trajectories when we consider MIX $_{c}$-MIX, MIX $c^{-}$CLA and CLA $_{c}$-CLA with a very large $c=10^{10}$ value. This extreme $c$ almost means that no constraint is imposed on the eigenvalue ratios of the scatter matrices. Therefore, these curves would essentially correspond to the traditional use of the BIC criteria (when using the MIX-MIX criterion) and the ICL (when using the MIX-CLA criterion). We can see that the constrained autMIXMIX procedure clearly outperforms traditional BIC and ICL criteria. Moreover, it appears that the gap between constrained and unconstrained curves seems to increase as the overlap increases and also if we increase the number of best possible solutions which are kept. Figure 9 also shows the average values of the above ARI over 100 simulations when the dimension of the simulated data sets is now increased to $p=6$. Although this higher dimensional case yields smaller ARI values than those obtained when $p=2$, we can see that the gap between constrained and unconstrained curves clearly increases in this new setting. Note also that very sensible ARI values are obtained, in spite of the higher problem dimensionality, when retaining the two and three best solutions returned from the proposed automated procedures. Finally, we can see that the observed differences from the application of the autMIXMIX, autMIXCLA and autCLACLA procedures are almost negligible here with $p=6$ case (especially in the central and right panels). The observed difference between the proposed methodology 
and the traditional use of the BIC and ICL (unconstrained) criteria is likely to increase with the dimension $p$ because spurious solutions are more likely to appear in these higher dimensional cases (see García-Escudero et al., 2014, 2015).

\section{Conclusions and further directions}

Three criteria for choosing the number of clusters in constrained model-based clustering have been proposed. Constraints make the associated (likelihood-based) target functions bounded and prevent the detection of non-interesting spurious solutions. Through our constraints we control the maximal ratio between the eigenvalues of the scatter matrices to be no greater than a fixed constant $c$, with $c \geq 1$. This constant serves to simultaneously control cluster departures from sphericity and heteroscedasticity among groups. In order to establish complexity-penalized criteria for choosing the number of clusters, we have taken into account the higher model complexity that a higher value of $c$ entails. In our opinion, clustering should not be seen as a fully automatic task providing just one single solution and any user has to play an active role by specifying somehow the desired type of partitions. This specification can be done by fixing $c$ depending on the clustering application. Additionally, a fully automated procedure producing a small and ranked list of optimal $(k, c)$ pairs has been proposed and illustrated in a simulated data set and in three well-known real data examples. Our approach provides a trade off between the degree of automation of the clustering process and the user attitude towards a black-box output. If the user is prepared to look at more than one sensible solution, our procedure is still fully automatic. We have also added a new graphical display which enables us to clearly appreciate what are the most important solutions together with their stability.

A simulation study has also been carried out in order to validate the performance of our proposed methodology. The results of this simulation study have shown the importance of including constraints and have pointed out the general superiority of our proposal to other non-constrained penalized likelihood approaches, such as the BIC and the ICL criteria. Moreover, although with a small degree of overlap among the groups our three constrained criteria seem to give approximately the same results, the autMIXMIX criterion generally outperforms the other two when the overlap increases. 
All the routines to obtain the results presented in this paper have been included in the FSDA toolbox for MATLAB which is freely downloadable from http://www.riani.it/MATLAB or from http://fsda.jrc.ec.europa.eu. More information about this routines can be found in the "online supplementary material". There are some other research lines that deserve to be explored in the future. For instance, it will be interesting to extend this methodology to other clustering problems, such as clusterwise linear regression or mixtures of factor analyzers. We are also investigating how to apply this approach in robust clustering. Specifically, we are interested in extending the complexity-penalized likelihood approach described in this paper within the TCLUST framework (García-Escudero et al., 2008), in order to choose $k$ and $c$ together with the trimming level $\alpha$. This is not an easy problem since these three parameters, $k, c$ and $\alpha$, are clearly interrelated. For instance, a high value of $\alpha$ could require a smaller $k$ given that some small clusters may be completely trimmed. Besides, a high value of $c$ may allow a certain fraction of background noise to be considered as an additional more scattered cluster and, thus, a higher $k$ may be be needed. Our feeling is that a reduced list of "sensible" $(k, c, \alpha)$ triplets, where the user can choose the robust cluster partition that better fits his/her purposes, can also be automatically derived in an analogous way to that in Section 5. Neykov et al. (2007) and Li et al. (2016) have already considered trimmed version of the BIC in clustering and mixture modeling problems.

\section{Supplemental Materials}

Matlab Code: The supplemental files for this article include Matlab programs which can be used to replicate the simulation study and the figures included in the article. File README contained in the zip file gives more details. (matlab_code.zip, zip archive)

Appendix: The supplemental files include the Appendix which gives the proof of Theorem 3.1 and a graphical illustration of it. Additional tables and figures are given for the material presented in Sections 3, 4 and 5. The application of the proposed methodology to the well-known "Iris data set" is also given. The three best ranked solutions for the real data set example are summarized by using functional boxplots. An explanation about how to use the developed routines to carry out the proposed 
methodology in the FSDA toolbox for MATLAB is also given (supplemental.pdf)

\section{Acknowledgments}

García-Escudero's and Mayo-Iscar's research was supported in part by the Spanish Ministerio de Economía y Competitividad and FEDER, grant MTM2014-56235-C2-1-P, and by Consejería de Educación de la Junta de Castilla y León, grant VA212U13 and VA002G18. Special thanks go to Anthony C. Atkinson, for helpful discussions and suggestions, and to Domenico Perrotta, for stimulating this research and for partially supporting it under the framework of the Automated Monitoring Tool (AMT) Project series. The authors thank the editor, the associate editor, and three anonymous referees for constructive comments.

\section{References}

Banfield, J. and Raftery, A. (1993). Model-based Gaussian and non-Gaussian clustering. Biometrics, 49:803-821.

Biernacki, C., Celeux, G., and Govaert (2000). Assessing a mixture model for clustering with the integrated completed likelihood. IEEE Trans. Pattern Anal. Mach. Intell, $22: 719-725$.

Bryant, P. (1991). Large-sample results for optimization-based clustering methods. $J$. Classif., 8:31-44.

Celeux, G. and Govaert, G. (1995). Gaussian parsimonious clustering models. Pattern Recogn., 28:781-793.

Day, N. (1969). Estimating the components of a mixture of two normal distributions. Biometrika, 56:463-474.

Forina, M., Armanino, C., Lanteri, S., and Tiscornia, E. (1983). Classification of olive oils from their fatty acid composition. In Martens, M. and Russwurm, H. J., editors, Food Research and data Analysis, pages 189-214. Applied Science Publishers, London. 
Fraley, C. and Raftery, A. (2002). Model-based clustering, discriminant analysis, and density estimation. J. Am. Stat. Assoc., 97:611-631.

Fraley, C. and Raftery, A. (2007). Bayesian regularization for normal mixture estimation and model-based clustering. J. Classification, 24:155-181.

Fraley, C., Raftery, A., Scrucca, L., Murphy, T., and Fop, M. (2017). mclust version 5.3 for R: Normal mixture modeling for model-based clustering, classification, and density estimation. available at https://cran.r-project.org/web/packages/mclust/.

Fritz, H., García-Escudero, L., and Mayo-Iscar, A. (2013). A fast algorithm for robust constrained clustering. Comput. Stat. Data Anal., 61:124-136.

García-Escudero, L. and Gordaliza (2005). A proposal for robust curve clustering. J. Classification, 22:185-201.

García-Escudero, L., Gordaliza, A., Matrán, C., and Mayo-Iscar, A. (2008). A general trimming approach to robust cluster analysis. Ann. Statist., 36:1324-1345.

García-Escudero, L., Gordaliza, A., Matrán, C., and Mayo-Iscar, A. (2015). Avoiding spurious local maximizers in mixture modeling. Stat. Comput., 25:619-633.

García-Escudero, L., Gordaliza, A., and Mayo-Iscar, A. (2014). A constrained robust proposal for mixture modeling avoiding spurious solutions. Adv. Data Anal. Classif., $8: 27-43$.

Hathaway, R. (1985). A constrained formulation of maximum likelihood estimation for normal mixture distributions,. Ann. Statist., 13:795-800.

Hennig, C. and Liao, T. (2013). How to find an appropriate clustering for mixed-type variables with application to socio-economic stratification,. J. Roy. Statist. Soc. Ser. C, 62:309-369.

Ingrassia, S. and Rocci, R. (2007). Constrained monotone EM algorithms for finite mixture of multivariate gaussians,. Comput. Stat. Data Anal., 51:5339-5351. 
Li, M., Xiang, S., and Yao, W. (2016). Robust estimation of the number of components for mixtures of linear regression models,. Computation. Stat., 31:1539-1555.

Maitra, R. (2009). Initializing partition-optimization algorithms. IEEE/ACM Trans. Comput. Biol. Bioinf., 6:1447-15.

Maitra, R. and Melnykov, V. (2010). Simulating data to study performance of finite mixture modeling and clustering algorithms. J. Comput. Graph. Stat., 19:354- 376.

McLachlan, G. and Peel, D. (2000). Finite Mixture Models. John Wiley Sons, Ltd.

McNicholas, P., ElSherbiny, A., Jampani, K., McDaid, A., Murphy, T., and Banks, L. (2015). pgmm: Parsimonious gaussian mixture models. available at https://cran.rproject.org/web/packages/pgmm/.

Milligan, G. and Cooper, M. (1985). An examination of procedures for determining the number of clusters in a data set. Psychometrika, 50:159-179.

Neykov, N., Filzmoser, P., Dimova, R., and Neytchev, P. (2007). Robust fitting of mixtures using the trimmed likelihood estimator. Comput. Stat. Data Anal., 52:299-308.

Riani, M., Cerioli, A., Perrotta, D., and Torti, F. (2015). Simulating mixtures of multivariate data with fixed cluster overlap in FSDA library. Adv. Data Anal. Classif., 9:2015.

Riani, M., Perrotta, D., and Torti, F. (2012). FSDA: a matlab toolbox for robust analysis and interactive data exploration,. Chemometr. Intell. Lab. Syst., 116:17-32.

Rousseeuw, P. (1987). Silhouettes: A graphical aid to the interpretation and validation of cluster analysis,. J. Comput. Appl. Math., 20:53-65.

Sun, Y. and Genton, M. G. (2011). Functional boxplots,. J. Comput. Graph. Stat., $20: 316334$.

Symons, M. (1981). Clustering criteria and multivariate normal mixtures. Biometrics, $37: 35-43$

Tibshirani, R., Walther, G., and Hastie, T. (2001). Estimating the number of data clusters via the GAP statistic. J. Roy. Statist. Soc. Ser. B, 63:411423. 